\title{
Short-Range Cooperation of Mobile Devices for Energy-Efficient Vertical Handovers
}

\author{
Xenofon Foukas, ${ }^{1,2}$ Kimon Kontovasilis $\mathbb{D}^{1},{ }^{1}$ and Mahesh K. Marina ${ }^{2}$ \\ ${ }^{1}$ Institute of Informatics \& Telecommunications, NCSR “Demokritos”, Agia Paraskevi, Greece \\ ${ }^{2}$ School of Informatics, The University of Edinburgh, Edinburgh, UK \\ Correspondence should be addressed to Kimon Kontovasilis; kkont@iit.demokritos.gr
}

Received 1 September 2017; Revised 8 December 2017; Accepted 19 December 2017; Published 28 January 2018

Academic Editor: Zhi Liu

Copyright (C) 2018 Xenofon Foukas et al. This is an open access article distributed under the Creative Commons Attribution License, which permits unrestricted use, distribution, and reproduction in any medium, provided the original work is properly cited.

\begin{abstract}
The availability of multiple collocated wireless networks using heterogeneous technologies and the multiaccess support of contemporary mobile devices have allowed wireless connectivity optimization, enabled through vertical handover (VHO) operations. However, this comes at high energy consumption on the mobile device due to the inherently expensive nature of some of the involved operations. This work proposes exploiting short-range cooperation among collocated mobile devices to improve the energy efficiency of vertical handover operations. The proactive exchange of handover-related information through low-energy short-range communication technologies, like Bluetooth, can help in eliminating expensive signaling steps when the need for a VHO arises. A model is developed for capturing the mean energy expenditure of such an optimized VHO scheme in terms of relevant factors by means of closed-form expressions. The descriptive power of the model is demonstrated by investigating various typical usage scenarios and is validated through simulations. It is shown that the proposed scheme has superior performance in several realistic usage scenarios considering important relevant factors, including network availability, the local density of mobile devices, and the range of the cooperation technology. Finally, the paper explores cost/benefit trade-offs associated with the shortrange cooperation protocol. It is demonstrated that the protocol may be parametrized so that the trade-off becomes nearly optimized and the cost is maintained affordable for a wide range of operational scenarios.
\end{abstract}

\section{Introduction}

Modern wireless networking is characterized by the availability of multiple radio access technologies, such as Wi-Fi (IEEE 802.11), 3G/4G mobile networks, and WiMAX (IEEE 802.16), with overlapping as well as complementary coverage areas. This diversity of available access opportunities, the proliferation of mobile devices equipped with multiple radio interfaces, and the impending emergence of next-generation (5G) mobile networks present a great opportunity for ubiquitous, always-on connectivity. A necessary ingredient along this way is a mechanism enabling mobile nodes (MNs) to seamlessly roam between heterogeneous wireless access networks, commonly referred to as the vertical handover (VHO). Such a mechanism could act as an enabler to a number of critical management operations, like mobility management and offloading.
There exist established standards, including 3GPP [1] and IEEE 802.21 [2], specifying multiradio mobility management frameworks for the realization of VHOs in a mediaindependent manner, that is, by abstracting the handover actions to isolate them from the details of the underlying radio access technologies. These frameworks include operations relevant to various handover-related aspects, including the issuance of handover triggers, the determination of a list of networks that are candidate handover targets, the selection of a target network among the candidates, and the execution of the handover (including related "book-keeping" operations) (see, e.g., [3]).

While the capability for VHOs undeniably leads to the enhancement of the overall service experience, it comes at a high cost for the mobile devices in terms of energy consumption, since some of the associated operations are inherently expensive. In the present world of battery-limited 
devices, where always-on availability is becoming increasingly important, there is a clear need for a more energy-aware multiradio mobility management framework.

With the above in mind, this paper aims at enabling energy-efficient vertical handovers. Towards this end, we propose a mechanism to exploit short-range cooperation among mobile devices as an optimization for improving the energy efficiency of VHO operations (Section 3). At the heart of the idea is the use of low-energy short-range communication technologies, like Bluetooth, for the periodic exchange of handover-related information between collocated MNs. This information is stored in a cache memory at each MN and is used once a VHO is triggered. The locality of the obtained information helps in reducing or in completely eliminating the number of expensive $\mathrm{VHO}$ operations, like scanning, effectively making the whole VHO more efficient.

Moreover, we develop a performance model that captures the costs and benefits of short-range cooperation, expressed in terms of average energy expenditure (Section 4). The model concisely encapsulates various cooperation and handover-related parameters like the radio link conditions in the area where the handover occurs, network loading conditions at candidate networks, the density of neighbors within the cooperation area, their tendency for associating with each of the candidate networks, and the range of the short-range communication technology. All these are important factors when considering handovers and the model manages to wrap them in concise yet descriptive closed-form expressions, also taking into account the cooperation cost. The descriptive power of the model is further demonstrated by considering a number of typical usage scenarios and developing customized versions of the modeling results for each of these (Section 5). The specialized expressions yield additional insight about the impact of various parameters to the mean energy expenditure of MNs.

We validate the modeling results via simulation and perform a study exploring the effect of the relevant parameters to the performance of the cooperation-assisted handover scheme by employing both simulations and the model and also involving a comparison with the conventional approach (Section 6). The results present several realistic scenarios in which the cooperation-assisted scheme is seen to outperform the conventional VHO approach. Through these results, we find that the most important factors influencing the efficiency of the cooperation-enabled mechanism are the average network load, the density of neighbors within the cooperation area, and their association preference with specific networks. Further results are presented regarding the cost and benefit trade-offs stemming from the shortrange cooperation protocol. It is shown that it is possible for this trade-off to become nearly optimized, with the cost remaining affordable for a wide range of operational scenarios through the proper parametrization of the protocol.

Along a more practical perspective, the paper also discusses implementation aspects of the short-range cooperation mechanism (Section 7), considering Bluetooth and Wi$\mathrm{Fi}$ as the enabling radio access technologies.
In terms of related work, there have been several works employing short-range cooperation as a means for optimization from various perspectives (e.g., [4-9]) but to the best of the authors' knowledge this is the first attempt to exploit this concept in the domain of VHO management. In the context of VHO management, closest in spirit to this paper is the work reported in [10], where geolocation information is exploited for improving quality of experience. However, [10] does not involve short-range cooperating peers and does not target energy efficiency. Regarding the modeling aspect of this paper, this is the first attempt at a comprehensive modeling of the energy costs associated with vertical handover mechanisms. The only other work bearing some relevance is [11], which investigates the energy requirements associated with the execution of VHOs. However, [11] addresses only conventional handover frameworks and proceeds on the basis of direct measurements on a prototype heterogeneous network testbed.

It is mentioned that this article is a significantly extended and enhanced version of the conference paper in [12]. The journal version provides several new contributions, including the formal treatment of typical usage scenarios and a discussion of additional insights arising from it, the extensive investigation of cost/benefit trade-offs related to the cooperation mechanism, and a consideration of protocol implementation aspects.

In the following section, we give a brief overview of the media-independent handover (MIH) operations and their associated energy efficiency bottlenecks, providing the conceptual basis for developing the paper's novel contributions described in subsequent sections.

\section{Media-Independent Handover Operations and Associated Bottlenecks}

To motivate our proposed short-range cooperation mechanism as an enabler of fast and energy-efficient handovers, we give a brief overview of conventional media-independent handover schemes according to the prevalent standards, such as 3GPP [1] or IEEE 802.21 [2]. While each vertical handover framework defines its own entities and a different set of actions and messages, the essence of the handover-related operations remains the same in all frameworks. Thus, the following review of important operations (from this paper's perspective) is expressed in generic terms and is confined to operations essentially relevant to this study.

Once a vertical handover is triggered, the $\mathrm{MN}$ queries an information service for a list of candidate networks, that is, networks serving the area where the $\mathrm{MN}$ is located and accessible by means of a radio access technology among those supported by the MN. While all such networks in the list are in principle candidates for becoming the target of the handover, there is no guarantee that all of them will actually be available to the $\mathrm{MN}$. One reason might be that the radio link to a candidate network might be poor due to bad positioning of the MN, despite the fact that the network covers the local area. Therefore, the $\mathrm{MN}$ needs to scan 
candidate networks to verify the link quality by measuring the received signal strength (RSS).

Furthermore, even in the case that a candidate network is accessible to the MN through a radio link of good quality, it must also have adequate resources to allocate in order for an association to be performed. Thus, apart from scanning, the MN needs to query the available networks for resources. If both of these operations are successful, the network can be chosen as a target for handover. Among the actions just outlined, there have been studies, for example, [11], showing that the scanning operation can be very costly in terms of energy even by an order of magnitude or more. Moreover, consulting the information service is an action that can incur latency to the whole vertical handover process, since such a service could be located in a server multiple hops away.

The generic operations just described can be combined in several ways to form a complete handover scheme. For example, the MN could scan all the candidate networks at once and then query those in which the scan was successful for resources. Figure 1 illustrates one such commonly employed scheme, referred to here as SCAN-FIRST. The scheme begins with the MN getting a list of the candidate networks by the information service. Each network is examined in turn by a scanning to check whether the radio link quality is good, followed immediately by a query for resources in case of a successful scan. The first network for which both operations succeed is chosen as the handover target, without checking the rest of the networks contained in the candidate networks list.

In the remainder of this paper, we will adopt SCANFIRST as a reference "conventional" scheme and as a basis for deploying the short-range cooperation-assisted enhancement to be proposed. However, it is equally possible to employ the proposed short-range cooperation assistance in conjunction with any alternative arrangement of the conventional handover base-operations. Correspondingly, the associated performance model of Section 4 (and the specialized forms in Section 5) can be readily adapted for such alternatives.

\section{Employing Short-Range Cooperation for Efficient VHOs}

The idea behind the short-range cooperation mechanism is that an $\mathrm{MN}$ does not wait for a handover trigger to occur to start collecting network-related information. Instead, it continually exchanges relevant information with peer MNs, employing a short-range wireless technology. This arrangement opens the possibility that some costly handover-related actions that would be mandatory in conventional mediaindependent VHO schemes, like scanning and consulting the information service, could be avoided when the need for a handover arises.

More specifically, each MN periodically broadcasts a notification about the network it is currently connected to (frequency, network name, radio access technology type, etc.) using a low-energy short-range communication interface (e.g., Bluetooth). Each listening MN gathers the information broadcast by its neighbors and updates a cache of candidate networks, so that it can be used later, once a VHO is initiated. The more short-term the cache is, the more accurate the information will be for the MN.

The effectiveness of such a mechanism greatly relies on the relevance of the obtained information. When the cooperation technology employed operates only within a short range (i.e., a few meters or at most tens of meters), it is highly likely (even though not guaranteed) that the network conditions observed by the peer MNs will also be very similar for most primary communication technologies that normally have a much larger range (e.g., Wi-Fi, LTE, or 5G NR). This locality of the obtained information could allow an $\mathrm{MN}$ to reduce or even completely eliminate the expensive $\mathrm{VHO}$ operations.

3.1. Selecting an Appropriate Short-Range Cooperation Protocol. Since the cooperation mechanism is employed for improving the energy efficiency of $\mathrm{VHO}$ operations, allowing the MNs to keep their short-range interfaces continuously active, constantly listening for broadcast information from collocated peers, would not be advisable, as it would lead to high energy consumption even in cases of energy-efficient technologies like Bluetooth low energy (BLE). It would thus be preferable if the MNs periodically deactivated their shortrange interfaces to save energy and activated them only in the case they needed to exchange context information. However, the energy savings of this approach come at the cost of a more difficult coordination among the cooperating nodes, because such coordination can occur only during the periods the MNs are active. Therefore, an asynchronous power-saving mechanism is required to allow the coordination among MNs in a simple manner and without the need of exchanging additional control messages.

There are several works on efficient cooperation mechanisms. However, most of them are cumbersome for our purpose. For example, [13] requires devices with GPS positioning and [14] needs a secondary radio interface for paging, while some works, for example, $[15,16]$, propose sophisticated broadcast mechanisms with high energy requirements.

The solution adopted in this work is based on [17], which proposes power-saving MAC protocols for information exchanges in IEEE 802.11-based ad hoc networks. Reference [17] proposes three protocols, namely, the dominatingawake-interval, periodically-fully-awake-interval, and quorum-based protocols, which allow mobile hosts to switch to a low-power sleep mode periodically. After assessing these protocols in terms of their energy efficiency, we chose for this work a slight modification of the periodically-fullyawake-interval protocol as it best matches our application setting and was shown to provide much better performance energy-wise compared to the other two [17].

The modification employed here over the original in [17] is that the protocol is targeted to operate above the MAC layer. The reason is that the same solution can be applied over different communication technologies without changing its basic concept. Another reason is to accommodate the possibility that the short-range communication interface 


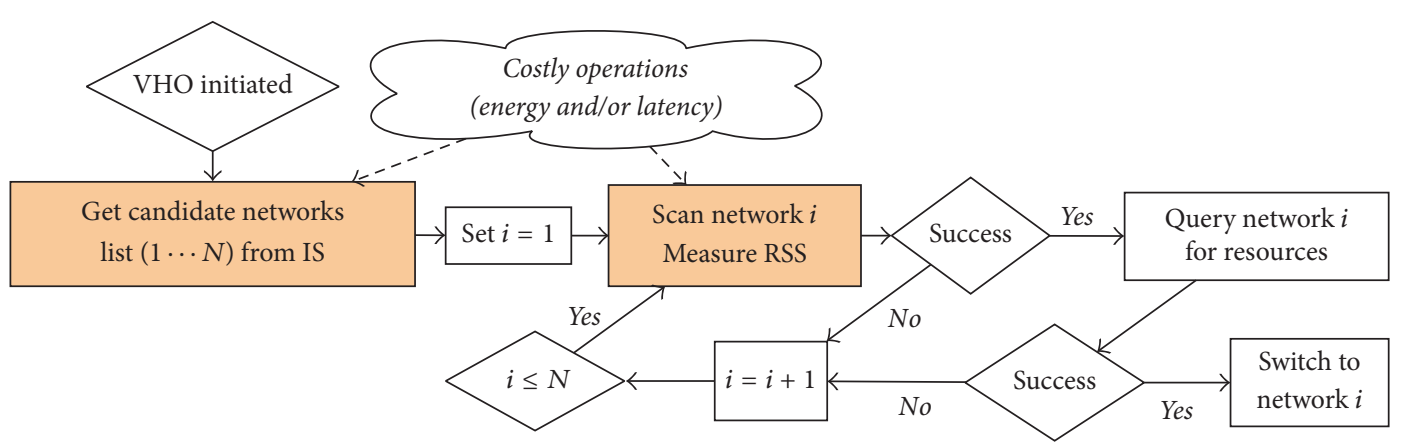

FIGURE 1: VHO procedure in the SCAN-FIRST scheme.

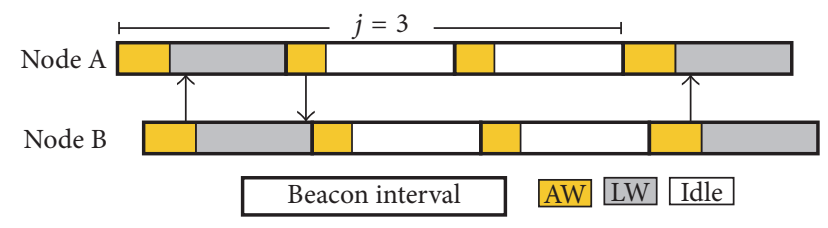

FIGURE 2: Modified periodically-fully-awake-interval protocol for the short-range cooperation between MNs. Neighbors exchange VHO-related information during AW and LW periods.

could also be used simultaneously by other processes for different operations. Optimizing the wireless technology at the MAC layer to work solely for the cooperation mechanism might have unpredictable and unwanted side effects for the rest of the processes. By applying the coordination protocols at a higher level, the cooperation mechanism can be used to save energy as intended, while allowing the operations of other processes to be performed unchanged (e.g., by using the short-range interface during the idle periods of the cooperation mechanism).

We now briefly review the main features of periodicallyfully-awake-interval. The discussion employs generic terms, avoiding MAC-related details unrelated to our context. According to the protocol, the exchange of information is performed using an asynchronous mode of operation. Each node operates using its own clock, without making any assumptions for the clocks of other MNs. Information exchanges occur in beacons, each lasting for a time equal to beacon interval (BI). Each $\mathrm{BI}$ is further subdivided in periods where the MN can transmit and receive context information from nearby MNs or remain idle. In the idle period, the shortrange interface is not used for information exchanges and could be turned off, set in a power-saving mode, or used for other operations irrelevant to the cooperation mechanism. The transmission of information lasts for a time equal to advertisement window (AW) and the reception for a time equal to listening window. Normally, beacons just transmit and stay idle for the rest of their duration. However, every $j$ th beacon is fully active and transmitting is followed by a listening phase. The parameters BI, AW, LW, and $j$ are global, applying to all MNs. This mechanism is illustrated in Figure 2 for two nodes and for $j=3$.
The scheme guarantees that a node will be heard by its neighbors within $j$ beacons regardless of how much their clocks drift away [17]. It is easy to see that, by using more low energy beacons than fully active ones, large idle periods can be obtained, during which little or no energy will be consumed by the short-range interface. The greater the value of $j$ is, the higher the energy savings from the idle periods become, at the expense of slower updates of handover-related information.

3.2. Exploiting the Information Exchanged through ShortRange Cooperation for Vertical Handovers. Once a VHO is triggered, the $\mathrm{MN}$ attempts to connect to a suitable candidate network among those suggested by its neighboring nodes, simply by consulting the information stored in its cache. Cache entries are tagged with a timestamp showing the time they were introduced in the memory and remain stored based on a threshold value showing how recent they are. The lower the threshold is, the faster an entry needs to be evicted from the cache. As long as the information held in the MN's cache is recent and comes from collocated MNs (due to the short-range technology employed for cooperation), the $\mathrm{MN}$ does not need to verify the radio links for networks in the cache and just queries each of the candidate networks in the cache for resources, without scanning first. If none of the networks in the cache has adequate resources to support connectivity, the $\mathrm{MN}$ falls back to the conventional $\mathrm{VHO}$ scheme and the list of candidate networks is checked by the procedure described in Section 2 after a preprocessing to remove networks for which a connection attempt has already been made. The steps performed by the MN when the shortrange cooperation mechanism is enabled are illustrated in Figure 3.

The way that the short-range cooperation mechanism operates can have multiple advantages over a conventional VHO scheme. The most important advantage is that the information exchanged using this approach can help in eliminating inherently expensive signaling steps, in terms of energy or latency, when the need for a VHO arises. More specifically, as already explained, since a short-range technology like Bluetooth is used, the information obtained will be accurate for the MN with a very high probability, allowing the omission of expensive scanning operations for ensuring network connectivity. Additionally, the MN can 


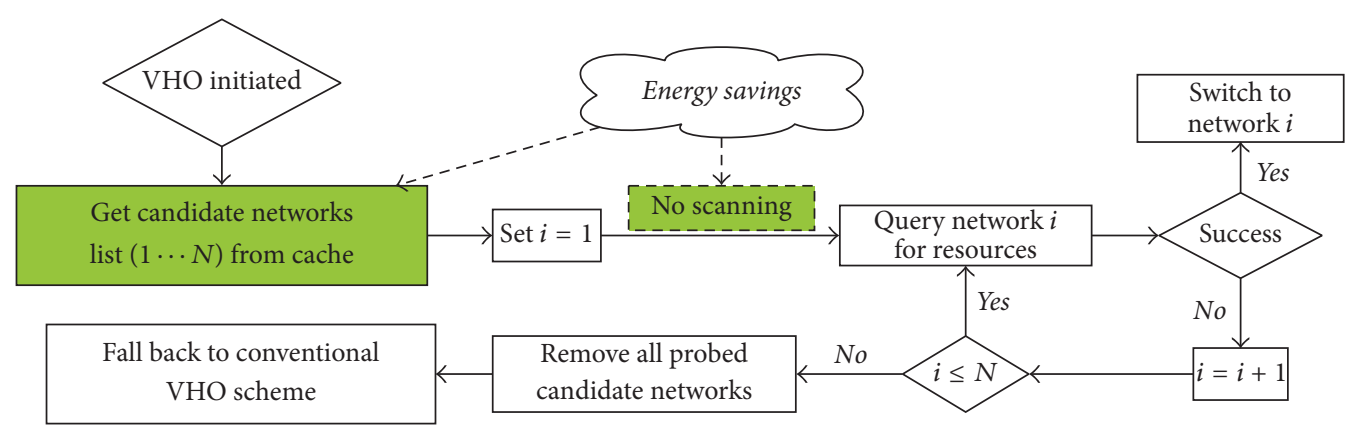

FIGURE 3: VHO procedure using the information obtained through the short-range cooperation mechanism.

attempt to perform the $\mathrm{VHO}$ using its cache entries, without consulting the information service, effectively reducing the handover latency. It should be noted that this improvement does not come with the price of a latency penalty for using the cooperation mechanism, since the coordination of MNs is a proactive process running constantly in the background.

\section{Model for Quantifying the Benefit of the Cooperation-Assisted VHO Mechanism}

We now develop a model to quantify the cost associated with the cooperation-assisted VHO mechanism in terms of the availability and status of the candidate networks, the node density in the cooperation area, and the radio link between these MNs and the candidate networks. Cost is quantified as the mean energy consumption experienced by an $\mathrm{MN}$ per handover. For the cooperation-assisted mechanism, the cost can be divided into two parts: the cost for the coordination and information exchanges of the MNs and the cost for performing the actual VHO procedure. For the conventional scheme, the only cost involved is that for performing the actual VHO procedure.

\subsection{Mean Cost for Performing Vertical Handovers}

4.1.1. Conventional Scheme. We first refer to the average cost per handover in the conventional scheme. We consider an observed $\mathrm{MN}$ for which the handover process is initiated. This MN needs to discover candidate networks and attempt to select a handover target among them. However, locally available candidate networks are not guaranteed to be accessible to the MN. We use a parameter $p$ (Summary of Model Parameters) to designate the probability that the observed $\mathrm{MN}$ will have a radio link of sufficient quality to a candidate network. The parameter $p$ characterizes location idiosyncrasies with respect to radio conditions, so it is assumed that the same parameter value applies to all candidate networks in the area. A $p$ close to 1 indicates that the area around the observed MN (and its short-range neighbors) is associated with good radio conditions, while low values of $p$ indicate poor radio conditions.

Even with good radio conditions, there is no guarantee that the observed MN will manage to associate with a given candidate network, since the network might not have adequate resources to allocate, due to network overload. To capture such loading considerations, we introduce a different parameter $q$ as the probability that a candidate network queried for resources towards becoming a handover target will respond positively. In the interest of capturing the overall impact of overloads versus normal loading conditions and of keeping the parameter space simple, a common value of $q$ is applied uniformly (but independently) to all candidate networks of the observed MN. Note that (in contrast to the relation of $p$ to the radio link conditions) values of $q$ close to 1 indicate low or moderate network load, while values closer to 0 indicate overload.

Given the link and load parameters just discussed, we now turn to the VHO operations performed in the conventional scheme (Figure 1). First, the observed MN needs to contact an information service in order to obtain a list of candidate networks. Then, the MN needs to scan each of these networks in turn to verify the link quality. If, along this process, a candidate network is successfully scanned, the MN will query this network for resources and will make it the handover target if the reply is positive, terminating the handover process. Each of these VHO-related operations incurs some cost for the MN. Let $C_{\mathrm{IS}}, C_{\mathrm{SCAN}}$, and $C_{\mathrm{Q}}$ be the cost for consulting the information service, for performing a scan, and for querying a network for resources, respectively.

Denote by $N$ the number of candidate networks available for the observed MN. In contrast to the other parameters discussed above, $N$ is an attribute of the observed $\mathrm{MN}$ alone. Different MNs subject to handover will in general be associated with a different number of candidate networks each. The total mean cost per handover will be equal to

$$
C_{\text {conv }}(N) \triangleq C_{\text {IS }}+\left(C_{\text {SCAN }}+p C_{\mathrm{Q}}\right) \frac{1-(1-p q)^{N}}{p q} .
$$

The terms in (1) encapsulate the cost of querying the information service and the mean cost for scanning and querying the $N$ candidate networks for resources, taking into consideration both successful and failed attempts through parameters $p$ and $q$. The proof of (1) can be found in the Appendix.

4.1.2. Short-Range Cooperation-Assisted Scheme. We now turn to the per handover cost for the short-range cooperation-assisted scheme. Again, $N$ candidate networks 
are assumed to be available for the observed MN subject to handover. However, while the $N$ networks are a priori available, the MN might not be aware of their existence. A given network among the candidates will be known to the $\mathrm{MN}$ only if at least one of its short-range neighbors is connected to it and has provided the relevant information to the $\mathrm{MN}$ in question. Therefore, in order to assess the probability with which a candidate network is known to the $\mathrm{MN}$, we need to model the existence of short-range neighbors.

For this, we draw upon the notion of a homogeneous spatial Poisson process as follows. The devices that can act as short-range peers are assumed to occur according to a spatial Poisson process of density $\sigma$ (expressed in devices per unit area) and the radio technology employed for the short-range cooperation is assumed to feature a communication range $r$, covering an area equal to $\pi r^{2}$. Then, the number of devices within range from the observed $\mathrm{MN}$ follows a Poisson process of rate

$$
\rho=\sigma \pi r^{2}
$$

Clearly, this rate encapsulates combined information about device density and about the range of the short-range communication technology.

Although the observed MN treats its $N$ a priori available candidate networks with equal preference, without any prioritization imposed, this symmetry does not necessarily propagate to the perception of its short-range cooperating peers. The set of candidate networks reflects the connectivity capabilities of the observed $\mathrm{MN}$ and these might be different from the capabilities of some of its peers. For example, the observed MN might support a subscription-based network in the area and this network may be unavailable to most of the MN's short-range cooperating peers. As a result, different candidate networks of an observed MN have a different likelihood of being used by its short-range cooperating peers. In the following, this peer-likelihood is also being referred to as a (peer-association) preference. Use of the term "preference" in this sense should not be taken to connote any form of prioritization when individual peers treat their own set of candidate networks.

The preferences of the short-range cooperating peers for the candidate networks available to the observed MN can be expressed through probabilities. Specifically, let $v_{i}, i=$ $0, \ldots, N, \sum_{i=0}^{N} v_{i}=1$, be the probabilities that a random cooperating peer would be attached to candidate network $i$. The parameter $v_{0}$, in particular, expresses the probability that peer MNs would be connected to a network not among the candidate networks of the observed MN, for example, a subscription-based network or a network for which the observed MN does not have the proper wireless interface. Note that, like $N$, the probabilities $v_{i}, i=0, \ldots, N$, are an attribute specific to the observed MN. Other MNs may be associated with a different set of $v_{i}$ values and a different value for $N$.

An a priori preference of a cooperating peer for a particular candidate network is not sufficient for associating with it. As was the case of the conventional VHO, the association presupposes the existence of an adequate radio link and the availability of network resources. In view of the relevant parameters $p$ and $q$, it follows that the probability with which a random cooperating peer will be associated with candidate network $i$ is equal to

$$
P_{i}=q p v_{i}, \quad i=1, \ldots, N
$$

Consequently, the peer will be connected to a network not among the candidates (or not connected at all) with probability

$$
P_{0}=1-\sum_{i=1}^{N} P_{i}=1-p q\left(1-v_{0}\right)
$$

Given the probabilities in (3) and (4) as well as the splitting property of the Poisson process, the counting processes relevant to the short-range neighbors of the observed $\mathrm{MN}$ connected to network $i$ occur as independent Poisson processes of rates $\rho P_{i}$.

As already discussed in Section 3, each MN has a cache in which the networks discovered through the short-range cooperation mechanism are stored. The observed node will have some network $i$ stored in its cache only if at least one of its discovered neighbors was connected to this network. In view of the Poisson structure just discussed, this event occurs with probability

$$
w_{i}=1-e^{-\rho P_{i}}, \quad i=1, \ldots, N
$$

and the associated indicator function of the event is $1_{i}$. The total number of networks for which information is available through the short-range cooperation mechanism will be $\aleph=$ $\sum_{i=1}^{N} 1_{i}$ with distribution

$$
\pi_{m} \triangleq \operatorname{Pr}\{\aleph=m\}, \quad m=0, \ldots, N
$$

Conditioned on the event $\aleph=m$, the MN will check the $m$ networks and successively try to connect to them. The associated conditional mean cost is denoted by $L_{c}(m)$. If the process just mentioned fails, the $\mathrm{MN}$ will fall back to the conventional VHO approach by consulting the information service and performing scans in order to discover the available candidate networks. In this case, from the $N$ candidate networks provided by the information service, the MN will only check the remaining $N-m$ networks that were not discovered by the short-range cooperation mechanism.

The conditional mean cost of checking the $m$ networks is

$$
\begin{aligned}
L_{c}(m) & =C_{\mathrm{Q}}\left[\sum_{l=1}^{m} l(1-q)^{l-1} q+m(1-q)^{m}\right] \\
& =C_{\mathrm{Q}} \frac{1-(1-q)^{m}}{q} .
\end{aligned}
$$

As this expression reflects, the mean cost includes the cost of successfully connecting to one of the $m$ networks or of failing to connect to any of them. Additionally, it can be observed that the only operation cost this expression includes 
is related to performing resource queries to the candidate networks. This is in accordance with the description of the cooperation-assisted VHO mechanism, in which scanning and consulting the information service are omitted until all networks discovered through the short-range mechanism have been investigated.

Using (6) and (7), the unconditional mean cost $L_{c}$ for inspecting and possibly connecting to some candidate using the short-range cooperation mechanism can be obtained as

$$
L_{c}=\sum_{m=0}^{N} \pi_{m} L_{c}(m)=C_{\mathrm{Q}} \frac{1-\phi(1-q)}{q},
$$

where $\phi(z)$ is the generating function

$$
\phi(z)=E z^{\aleph}=\prod_{i=1}^{N} E z^{1_{i}}=\prod_{i=1}^{N}\left(1-w_{i}+w_{i} z\right) .
$$

Moreover, by recalling that the check of the $m$ candidates fails with probability $(1-q)^{m}$ and by employing (1), one can readily calculate the mean cost associated with the "backup" conventional VHO scheme, employed after failing to connect to $m$ networks recently used by neighboring nodes. Specifically,

$$
\begin{aligned}
L_{r} & =\sum_{m=0}^{N} \pi_{m}(1-q)^{m} C_{\text {conv }}(N-m)=C_{\mathrm{IS}} \phi(1-q) \\
& +\left(C_{\mathrm{SCAN}}+p C_{\mathrm{Q}}\right) \\
& \cdot \frac{\phi(1-q)-(1-p q)^{N} \phi((1-q) /(1-p q))}{p q} .
\end{aligned}
$$

The average total cost for performing the handover is $C_{\text {coop }}=L_{c}+L_{r}$, so by combining (8) and (10), we get

$$
\begin{aligned}
& C_{\text {coop }}=C_{\mathrm{IS}} \phi(1-q)+C_{\mathrm{Q}} \\
& \cdot \frac{1-(1-p q)^{N} \phi((1-q) /(1-p q))}{q}+C_{\mathrm{SCAN}} \\
& . \frac{\phi(1-q)-(1-p q)^{N} \phi((1-q) /(1-p q))}{p q}
\end{aligned}
$$

Typically, $C_{\text {SCAN }}$ dominates the other cost constants by an order of magnitude. This observation, together with the fact that when the number of candidate networks $N$ is not too small $(1-p q)^{N} \ll 1$, leads to a simplification of the exact form (11), expressed by the approximation

$$
C_{\mathrm{coop}} \approx C_{\mathrm{SCAN}} \frac{\phi(1-q)}{p q} \approx C_{\mathrm{conv}} \phi(1-q)
$$

using also (the approximate form of) (1). Expression (12) links the mean cost of the cooperation-assisted VHO mechanism (excluding the cost for the information exchanges) to the mean cost for the conventional VHO approach. The improvement factor is seen equal to $\phi(1-q)<1$.
4.2. Energy Consumption for Mobile Node Coordination. Let $T$ be the average time between two consecutive VHOs. Also, let $E_{\mathrm{AW}}, E_{\mathrm{LW}}$, and $E_{\mathrm{IDLE}}$ be the total energy consumed by the mobile node during an AW, LW, or idle period, respectively. $E_{\mathrm{IDLE}}$ could be set either to a low value, if the interface used for short-range cooperation has entered a power saving mode, or to zero if it is completely deactivated.

As already discussed in Section 3.1, the coordination scheme employs one fully active beacon every $j$ beacons. This means that one round of this scheme is completed after time equal to $j \mathrm{BI}$ and thus the total number of rounds performed in the interval between two consecutive VHOs will be $T / j \mathrm{BI}$ on average. Moreover, it is easy to see from Figure 2 that the energy required for one round will be $j E_{\mathrm{AW}}+E_{\mathrm{LW}}+(j-1) E_{\text {idle }}$. Therefore, the total energy, $E_{\text {coord }}$, spent on average by the coordination mechanism between two VHOs will be

$$
E_{\text {coord }}=\left[j E_{\mathrm{AW}}+E_{\mathrm{LW}}+(j-1) E_{\mathrm{IDLE}}\right] \frac{T}{j \mathrm{BI}} .
$$

\section{Typical Usage Scenarios}

In this section, the descriptive power of the model is demonstrated by investigating how the conditions related to the likelihood of short-range peers for associating with the various candidate networks of the observed MN (i.e., the probabilities $v_{i}$ ) would affect the mean energy consumption of the observed MN when performing VHOs. As mentioned in the previous section after (12), the improvement to the energy consumption-related costs offered by the cooperation-assisted mechanism is expressed through the factor $\phi(1-q)$. Thus, we investigate the effects associated with various realistic scenarios by means of the corresponding forms for the generator (9).

5.1. The Candidate Networks for the Observed MN Are Equally Accessible to Neighbors. In this scenario, it is assumed that short-range peers would prefer the candidate networks almost equally likely. This could represent a typical scenario in which all the wireless networks available in an area use only well-known technologies (e.g., 802.11, LTE, UMTS, etc.), which are supported by most mobile devices and are not subscription-based. We still allow the possibility that shortrange peers might also connect to networks other than the observed MN's $N$ candidate networks, so, in general, $v_{0}$ might be nonzero. In accordance with these observations, $v_{i}=(1-$ $\left.v_{0}\right) / N, \forall i=1 \cdots N$. For this set of parameters, (5) yields

$$
w_{i}=1-e^{-\rho p q\left(1-v_{0}\right) / N}, \quad i=1, \ldots, N
$$

and (9) simplifies to

$$
\phi(z)=\left[e^{-\rho p q\left(1-v_{0}\right) / N}+\left(1-e^{-\rho p q\left(1-v_{0}\right) / N}\right) z\right]^{N} .
$$

Furthermore, if $N$ is sufficiently high,

$$
\begin{aligned}
\phi(z) & \approx\left[1-\frac{\rho p q\left(1-v_{0}\right)}{N}(1-z)\right]^{N} \\
& \approx e^{-\rho p q\left(1-v_{0}\right)(1-z)}
\end{aligned}
$$


which expresses the fact that, for sufficiently many candidate networks, the value of $\phi(z)$ becomes insensitive to the number of such networks, depending only on the device density and cooperation range (expressed through the rate $\rho$ of the Poisson process in (2)), on the radio conditions and the network load status, through $p$ and $q$, respectively, and on the likelihood $v_{0}$ of peers connecting to noncandidate networks.

This (asymptotic) invariance to the number of candidate networks can be intuitively explained, considering that the observed $\mathrm{MN}$ will probably manage to connect to one of the first few networks discovered using the short-range cooperation approach and thus no matter how many networks were actually discovered, no attempt of querying for resources or connecting will be made to most of them.

In connection with the energy consumption improvement (12), substituting $z=1-q$ to (16) yields

$$
\phi(1-q) \approx e^{-\rho p q^{2}\left(1-v_{0}\right)}
$$

which shows that the network load has a greater impact on the efficiency of the cooperation-assisted VHO mechanism than the link quality (quadratic effect of $q$ compared to linear effect of $p$ in the exponent of (17)). Similar remarks apply also to the other usage scenarios considered later in this section.

5.2. Candidate Networks of Varying Accessibility. In this scenario, we assume that there are two types of wireless networks: those which are likely to be preferred, corresponding to a high probability $v_{h}$, and those that are unlikely, corresponding to a small probability $v_{s}$. Typical scenarios in which such conditions might occur would be the simultaneous existence of multiple 802.11 networks in the same area, some of which would be completely free and some of which would incur a charge to the user or cases in which the observed $\mathrm{MN}$ has some kind of interface that is rare in some area (e.g., a European mobile device in a country having WiMAX networks).

Let $h$ be the number of likely preferred networks and let $N-h$ be the number of unlikely networks. Since $v_{s}$ is small, the probability $v_{h}$ becomes

$$
v_{h}=\frac{1-v_{0}-(N-h) v_{s}}{h} \approx \frac{1-v_{0}}{h} .
$$

Application of (5) now leads to

$$
\begin{aligned}
& w_{h}=1-e^{-\rho p q v_{h}} \approx 1-e^{-\rho p q\left(1-v_{0}\right) / h}, \\
& w_{s}=1-e^{-\rho p q v_{s}} \approx \rho p q v_{s} .
\end{aligned}
$$

Using these results and the fact that $v_{s} \ll 1$, (9) becomes

$$
\begin{aligned}
\phi(z) \approx & {\left[1-\rho p q v_{s}(1-z)\right]^{(N-h)} } \\
& \times\left[e^{-\rho p q\left(1-v_{0}\right) / h}+\left(1-e^{-\rho p q\left(1-v_{0}\right) / h}\right) z\right]^{h} \\
\approx & {\left[1-\rho p q v_{s}(1-z)(N-h)\right] } \\
& \times\left[e^{-\rho p q\left(1-v_{0}\right) / h}+\left(1-e^{-\rho p q\left(1-v_{0}\right) / h}\right) z\right]^{h} .
\end{aligned}
$$

Since $v_{s} \ll 1$, the first factor of this expression is close to 1 and thus $\phi(z)$ becomes approximately equal to the second factor in (20). Comparison with (15) reveals that this factor is equal to the value of the generating function $\phi(z)$ that would result if only the $h$ highly likely networks were available. Once more, this is an intuitive result, since the small probability $v_{s}$ makes the discovery of cooperating nodes connected to unpopular networks improbable. As the first factor in (20) indicates, the $N-h$ unpopular networks still contribute somewhat to the energy efficiency of the cooperation-assisted VHO mechanism (expressed through a further reduction of the value of $\phi(z)$ below 1), but this contribution is minor compared to the effect from the $h$ preferred networks.

5.3. Areas with Wireless Networks Not Supported by the Observed MN. In this case, it is assumed that most of the networks popular among the short-range peers in the coverage area either are subscription-based or are using a technology for which the observed $\mathrm{MN}$ does not have a proper interface. In such a scenario, we assume that $v_{0}$ is close to 1 ; thus, $1-v_{0}$ is small. Furthermore, for each $i=1, \ldots, N$, one has $v_{i}<\sum_{j=1}^{N} v_{j}=1-v_{0}$, so all other $v_{i}$ are small too and (5) becomes

$$
w_{i}=1-e^{-\rho p q v_{i}} \approx \rho p q v_{i}
$$

By combining this result with (9) and with the fact that $\prod_{j}(1-$ $\left.\delta_{j}\right) \approx 1-\sum_{j} \delta_{j}$, whenever $\left|\delta_{j}\right| \ll 1$, one obtains

$$
\begin{aligned}
\phi(z) & \approx 1-\rho p q(1-z) \sum_{i=1}^{N} v_{i} \\
& =1-\rho p q\left(1-v_{0}\right)(1-z) .
\end{aligned}
$$

The invariance of this result to $N$ supports the intuition that when most short-range peers of the observed MN prefer networks different than the candidate networks, it becomes unlikely for the MN to discover and exploit the candidate networks, regardless of their number. Most probably, the $\mathrm{MN}$ will need to fall back to the conventional mechanism. Indeed, using (22), one sees that the energy consumption improvement factor in (12) now becomes $\phi(1-q) \approx 1-$ $\rho p q^{2}\left(1-v_{0}\right)$, and this is close to unity (regardless of network load and radio link conditions) because $1-v_{0}$ is small.

It is noted that the right-hand side of (22) can also be obtained by employing (16) and noting that $1-v_{0}$ is small. However, result (22) is stronger, because it does not presuppose a sufficiently high $N$ or equally preferred candidate networks.

\section{Validation and Performance Evaluation}

6.1. Model Validation via Simulation. To validate the model, we employed a customized version of the well-known ns-3 simulator, enhanced with cooperation-assisted VHO capabilities. The cooperation mechanism was implemented in the form of an application running on top of the MAC layer of the MNs and operating according to the protocol 
described in Section 3. Short-range communications between neighboring devices were confined within a range of $r=$ $15 \mathrm{~m}$. The duration of a beacon and the interval between two fully active beacons were set equal to $\mathrm{BI}=3.2 \mathrm{~s}$ and $j=12$, respectively. The duration of the advertisement window (AW) within a beacon was set to $10 \%$ of $\mathrm{BI}$, that is, $0.32 \mathrm{~s}$. The costs of individual $\mathrm{VHO}$-related operations were set based on the measurements in [11] $\left(C_{\mathrm{Q}}=0.02 \mathrm{~J}, C_{\mathrm{SCAN}}=8.6 \mathrm{~J}\right.$, and $C_{\mathrm{IS}}=$ $0.02 \mathrm{~J})$, while the power consumption for transmission and reception through the short-range technology was set based on the Bluetooth measurements in [18] $\left(E_{\mathrm{AW}}=0.08 \mathrm{~J}\right.$ and $E_{\mathrm{LW}}=0.07 \mathrm{~J}$, resp.). It was also assumed that, during the idle period, the short-range interface is disabled; thus $E_{\mathrm{IDLE}}=0$.

The simulation scenarios under study involved 10 different Wi-Fi networks in an area where short-range communications and handover operations were captured in a square grid of $300 \times 300 \mathrm{~m}^{2}$. All of the involved wireless networks employed the default parameters of ns-3 (log distance propagation model [19], Tx power, and energy detection threshold [20]), resulting in a range of about $150 \mathrm{~m}$. As a result, all of the networks effectively acted as candidate networks, while the MNs were always within the coverage of at least one (but possibly more) wireless network.

The networks were divided into 2 types: $N_{\mathrm{A}}=3$ networks were of type $A$, representing networks offering premium services; the remaining $N_{\mathrm{B}}=7$ were of type $\mathrm{B}$, representing open-access networks. Networks of both types were deployed uniformly in the square grid. The population of MNs was also heterogeneous: $\alpha_{\mathrm{I}}=10 \%$ of the participating MNs were assigned to the device class I and the remaining $1-\alpha_{\mathrm{I}}$ to the device class II. The MNs of class I were capable of associating with all $N_{\mathrm{A}}+N_{\mathrm{B}}$ networks, while the MNs of class II could associate only with the $N_{\mathrm{B}}$ open-access networks. It should be mentioned that devices of class I were assumed to have no preference in associating with candidate networks of type $\mathrm{A}$ or B; that is, networks were not ordered based on their classes.

Various device densities were considered for multiple use cases from rural environments with only a few devices operating in a wide area to dense-urban areas. The number of MNs participating in a simulation run was calculated by multiplying the device density parameter value of the scenario under study to the area of the simulation grid. The evaluated densities were based on the density measurements in [21]. With respect to mobility and since the short-range cooperation mechanism is mostly affected by the average nodal density without regard for the exact mobility pattern, we employed the standard random waypoint mobility model already available in the ns-3 simulation platform. The MNs could freely move in and out of the area under study with random speeds of up to $10 \mathrm{~m} / \mathrm{s}$. It is noted that the mean time between VHOs was not set to a static, arbitrary value. Instead, it was determined through preliminary simulation runs for each scenario considered by measuring the number of $\mathrm{VHO}$ experienced by mobile nodes in a predefined amount of time. In this setup, a $\mathrm{VHO}$ was triggered once an $\mathrm{MN}$ missed a consecutive number of beacons from the base station it was associated with (this miss signifying motion out of the network's coverage area).
In order to properly match the parameters of the analytical model to the simulation setup, the network preferences $v_{i}$ of the short-range cooperating peers were set, depending on the class of MNs they refer to, according to the number and type of networks, previously mentioned. Specifically, MNs of class I have access to all $N_{\mathrm{A}}+N_{\mathrm{B}}$ networks, so they are associated with a set of probabilities $v_{i}^{\mathrm{I}}, i=0, \ldots, N_{\mathrm{A}}, N_{\mathrm{A}}+$ $1, \ldots, N_{\mathrm{A}}+N_{\mathrm{B}}$. Of these, the indices $i=1, \ldots, N_{\mathrm{A}}$ refer to the probability with which a random short-range peer (which may belong to any of the MN class I or II) may associate with the indexed network of type $A$. The remaining indices $i=N_{\mathrm{A}}+1, \ldots, N_{\mathrm{A}}+N_{\mathrm{B}}$ refer to random peers' association with networks of type B. Correspondingly, MNs of class II are associated with a set of probabilities $v_{i}^{\mathrm{II}}, i=0, \ldots, N_{\mathrm{B}}$, where indices $i=1, \ldots, N_{\mathrm{B}}$ refer to random peers' association with networks of type $B$.

For the devices of class I, the $N_{\mathrm{A}}$ networks of type A can only be discovered through neighbors that also belong to class I. Each of these neighbors would prefer to associate with any of the $N_{\mathrm{A}}$ networks with equal probabilities $1 /\left(N_{\mathrm{A}}+N_{\mathrm{B}}\right)$ and since these MNs form $\alpha_{\mathrm{I}}$ of the total population, the probabilities $v_{i}^{\mathrm{I}}, i=1 \cdots N_{\mathrm{A}}$, become $\alpha_{\mathrm{I}} /\left(N_{\mathrm{A}}+N_{\mathrm{B}}\right)=0.01$. For the remaining $N_{\mathrm{B}}$ networks of type $\mathrm{B}$, each $\mathrm{MN}$ assigned to class I would have a preference of $1 /\left(N_{\mathrm{A}}+N_{\mathrm{B}}\right)$, while each $\mathrm{MN}$ assigned to class II would have a preference of $1 / N_{B}$. As a result, the probability $v_{i}^{\mathrm{I}}, i=N_{\mathrm{A}}+1, \ldots, N_{\mathrm{A}}+N_{\mathrm{B}}$, becomes $\alpha_{\mathrm{I}} /\left(N_{\mathrm{A}}+N_{\mathrm{B}}\right)+\left(1-\alpha_{\mathrm{I}}\right) / N_{\mathrm{B}} \approx 0.139$. Based on this, it follows that $v_{0}^{\mathrm{I}}=1-\sum_{i=1}^{N_{\mathrm{A}}+N_{\mathrm{B}}} v_{i}^{\mathrm{I}}=0$. Using similar arguments to that of the previous case, the probability $v_{j}^{\mathrm{II}}, j=1, \ldots, N_{\mathrm{B}}$, becomes equal to $\alpha_{\mathrm{I}} /\left(N_{\mathrm{A}}+N_{\mathrm{B}}\right)+\left(1-\alpha_{\mathrm{I}}\right) / N_{\mathrm{B}} \approx 0.139$ and therefore $v_{0}^{\mathrm{II}}=1-\sum_{j=1}^{N_{\mathrm{B}}} v_{j}^{\mathrm{II}}=0.03$.

The final parameters required for the comparison of the model results to the simulations are the link quality $p$ and the network load $q$. These parameters are inherent to the scenario under study and therefore again matched to the simulation setting. The value of parameter $p$ was obtained through the following methodology: a simulation run was performed for $300 \mathrm{~s}$ with $100 \mathrm{MNs}$ using the conventional VHO scheme. During this period, the total number of handover requests was recorded for each $\mathrm{MN}$ along with the number of handover request timeouts due to no reply from the network. The parameter $p$ was then calculated by averaging the ratio of timeouts to the total number of handover attempts for all the MNs. The idea behind this approach is that the reason of the timeout was that handoverrelated messages were lost by the $\mathrm{MN}$ or the network due to bad signal quality, while the MN was moving. Parameter $p$ was found to be equal to approximately 0.4 , corresponding to an average link quality.

The value of parameter $q$ was calculated in a similar manner with the difference that instead of recording the number of handover request timeouts, we recorded the rejection messages sent by the candidate networks to association requests due to no resources being available. While the parameter $p$ depends on the simulation topology, $q$ depends on the network resources that can be allocated to the associated MNs. Therefore, in order to simulate different 
mean network loads, we varied the number of MNs that each network could accept, with higher thresholds leading to lower mean network loads and vice versa. Using this methodology, we managed to achieve low, medium, and high network loads corresponding approximately to a value of $0.85,0.5$, and 0.15 for the model parameter $q$.

Based on this methodology, we performed simulations with various device densities and network loads for both classes of MNs. The results can be seen in Figures 4(a) and 4 (b) in comparison to the results of the analytical model when using the parameters derived from the simulation setting. It is noted that the results for the cooperation-assisted $\mathrm{VHO}$ include both the energy spent for the short-range cooperation protocol and the energy for selecting a handover target. As it can be observed, the simulations validate the model with both approaches giving very similar results for all the cases under study. Moreover, it can be seen that the cooperationassisted scheme can be more energy-efficient compared to the conventional scheme in several interesting cases, becoming worse only in rural areas, where the discovery of neighboring nodes would be a rare event.

On a second look, we can also observe that the mean energy consumed per handover and the average network load follow similar trends, with high network loads leading to much higher energy consumption for both classes of devices. Moreover, it can be seen that higher network loads can negate the benefits of the cooperation-enabled mechanism even in very dense environments. This is because handover attempts will fail with a very high probability, regardless of the number of networks discovered through cooperation, and therefore a larger number of networks will need to be queried for resources, increasing the mean energy consumption. Moreover, we can observe that devices of class II have lower mean energy consumption compared to devices of class I in all cases, with the gap increasing as the network load increases. This is intuitive, since devices of class I have a wider range of networks to choose from and therefore they will spend more energy on average, while querying a larger number of candidate networks. As a final remark, we can see that using a global "average" value of $q$ works well, even in setups with multiple network classes, as the one simulated. It is worth mentioning that the conventional scheme yields the same energy consumption results regardless of the scenario, since the only thing that changes is the nodal density, which for the conventional case is an irrelevant factor.

We now proceed in a further analysis of the energy requirements of the cooperation-assisted scheme through the energy breakdown illustrated in Figures 4(c) and 4(d). We can observe that, in both cases, the biggest energy cost of this scheme comes from the actual VHO process. The energy cost for the short-range communication is much smaller and varies from only $5 \%$ up to $17 \%$ of the total cost depending on the scenario under study (network load and radio signal quality). It should be noted that the short-range communication cost is constant for both class I and class II MNs (about $4 \mathrm{~J} / \mathrm{VHO}$ ). This is because the parameters of the short-range protocol were configured in an identical way for both classes of MNs, while at the same time the mobility pattern employed led to almost the same total number of VHOs for both cases.

The impact of the short-range communication can also vary depending on the mobility of the MNs and on the configuration of the beacon interval. This is illustrated in the simulation results of Figure 5, where we varied the periodicity of fully active beacons (parameter $j$ ) as well as the average time between two VHOs $T$ (by manipulating the speeds of the MNs). Note that the values displayed in the figure represent the total energy expenditure per handover, including the overhead of the short-range cooperation and the effect of "cache misses" leading to the invocation of the more expensive conventional VHO scheme.

We can observe that when MNs perform VHOs more frequently (smaller $T$, due to higher mobility), they consume a smaller amount of energy per handover. This is because the network information obtained through the cooperation mechanism is exploited more frequently at a smaller per handover cost (due to the fact that the cooperation energy overhead depends linearly on $T$; see (13)). As a result, the cooperation benefit to cost ratio grows larger with higher handover frequencies.

Orthogonally to this effect, we can also see that as the period $j$ between two fully active beacons increases, the energy drastically drops up to a point and then remains almost constant for a while and, ultimately, increases again (at a rate much smaller than that of the initial drop). The initial big drop in the energy consumption comes from the savings that the MNs get through the reduction in the frequency of advertising network information to their peers. The energy increase trend as $j$ grows very large (and active beacons become sparse) occurs because it becomes more difficult for the MNs to discover networks through the cooperation mechanism. This forces them to fall back to the conventional scheme more frequently and thus results in higher energy consumption. Finally, the fact that the energy per handover remains almost constant for intermediate values of $j$ indicates that the efficiency of the short-range cooperation mechanism is relatively insensitive to the exact value of $j$ (within a range).

Based on this analysis, we can see that although choice of the optimal $j$ depends on the operational environment, it is readily possible to determine a nearly optimal value. Indeed, as evidenced from the results in Figure 5, setting this parameter to a value close to 20 provides a good tradeoff between energy consumption and discovery of networks through the short-range cooperation mechanism for a broad range of operational conditions.

A final important aspect of the short-range cooperation is related to the cost of the mechanism in terms of the short-range radio access technology spectrum utilization. This mechanism can be seen as a process that utilizes the spectrum in a TDM fashion, where the radio resources of the short-range technology can be used by other applications during idle periods. Based on Figure 2, it may be seen that the fraction of time the short-range communications channel remains idle is equal to $(1-1 / j)(1-\mathrm{AW} / \mathrm{BI})$. By employing the values of BI and AW used in the previously presented simulation results, Table 1 displays the percentage 


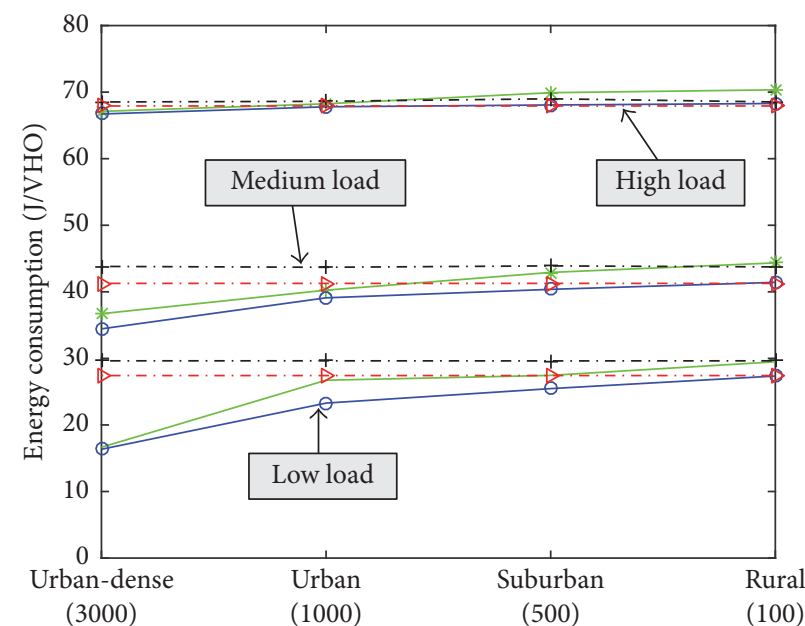

Environments with decreasing device density (neighbors $/ \mathrm{km}^{2}$ )

$\longrightarrow$ Short-range (simulation) $\rightarrow-$ Conventional (model)

- - Short-range (model)

+- Conventional (simulation)

(a) MNs in class I
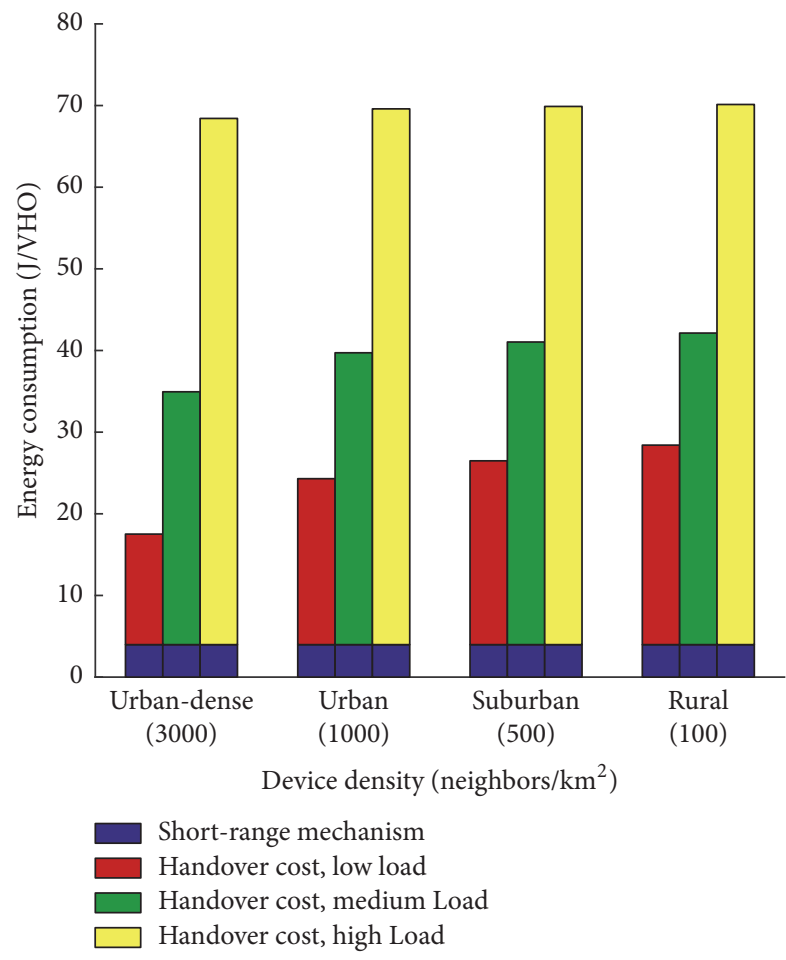

(c) MNs in class I

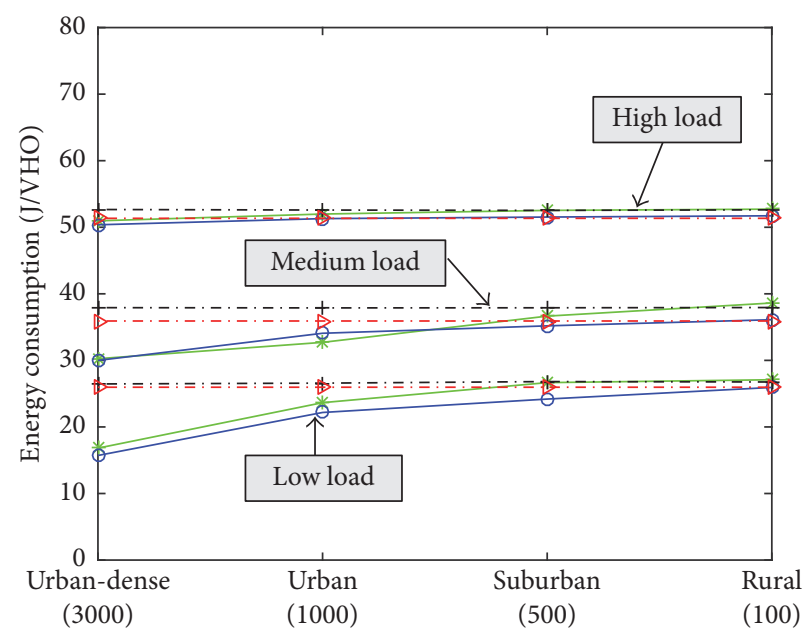

Environments with decreasing device density (neighbors $/ \mathrm{km}^{2}$ )

*- Short-range (simulation) $\rightarrow-$ Conventional (model)

$\rightarrow$ Short-range (model)

+- Conventional (simulation)

(b) MNs in class II
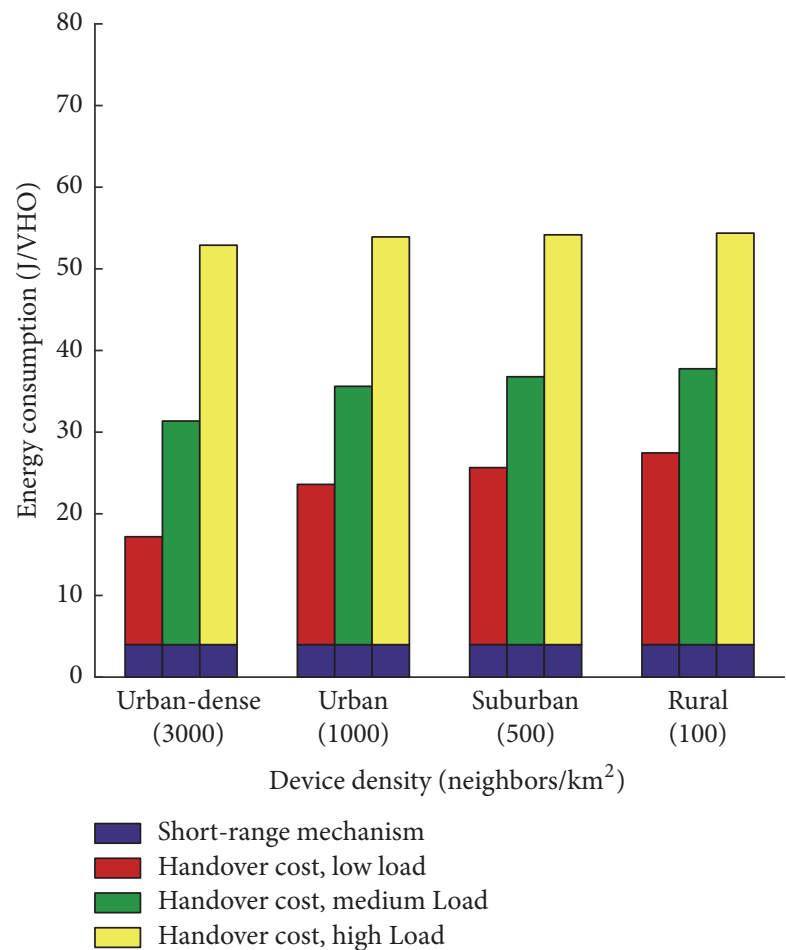

(d) MNs in class II

FIGURE 4: ((a) and (b)) Simulation and model-based average energy consumption of the cooperation-assisted VHO mechanism compared to that of the conventional VHO scheme for various network loads and device densities. ((c) and (d)) Energy breakdown of cooperation-assisted VHO mechanism.

of idle time corresponding to various values of $j$. As we can observe, for all the practical scenarios from Figure 5, the short-range technology remains idle for more than $82 \%$ of the total time, allowing other applications that depend on short-range communications to coexist over the same mobile device.
6.2. Further Numerical Results Based on the Model. Having validated the model developed in Section 4, we can now use it to expand our evaluation of the cooperation-enabled VHO mechanism. For the results in this subsection, we assume that all MNs belong to the same class and that all candidate networks are of the same type. Moreover, the average time $T$ 


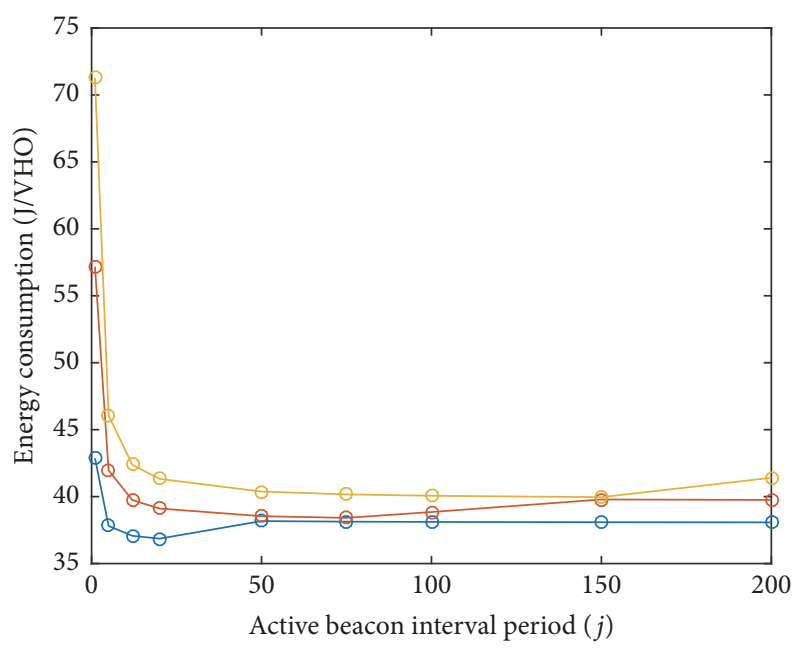

Average time between VHOs (s)

○ $T=100.3$

○- $T=301.87$

$\frown T=500.45$

FIGURE 5: Impact of short-range cooperation mechanism configuration to the overall energy consumption.

TABLE 1: Consumption of short-range technology spectrum resources for cooperation-assisted VHO mechanism.

\begin{tabular}{lc}
\hline Active beacon interval period $(j)$ & Idle time $(\%)$ \\
\hline 12 & 82.5 \\
20 & 85.5 \\
100 & 89.1 \\
200 & 89.6 \\
\hline
\end{tabular}

between consecutive handovers is set to $300 \mathrm{~s}$ and the device density is fixed to $\sigma=0.003$ neighbors $/ \mathrm{m}^{2}$ corresponding to a dense-urban area. The link quality and network load parameters $p$ and $q$ are adjusted according to the evaluation scenario under study, while the remaining parameters of "Summary of Model Parameters" are kept the same as the ones described in Section 6.1.

We begin by evaluating the energy consumption of the cooperation-assisted $\mathrm{VHO}$ mechanism and comparing it to that of the conventional scheme described in Section 2. The evaluation is made in terms of the number $N$ of available candidate networks in the area of the $\mathrm{MN}$ and of the various network preferences of its neighbors, expressed though the values of $v_{i}$. More specifically, we examine the performance of the cooperation-assisted VHO scheme in an area with networks of low average load $(q=0.9)$ and good link quality $(p=0.8)$ for three scenarios. The first scenario (I in Figure 6(a)) corresponds to a setting where the short-range neighbors of the observed $\mathrm{MN}$ exhibit comparable preference to the candidate networks available to the observed $\mathrm{MN}$. This corresponds to the setting discussed in Section 5.1 and is modeled by $v_{i}=1 / N, i=1, \ldots, N$, and $v_{0}=0$. As already discussed in Section 5.1, this could be a typical scenario in which all the wireless networks available in an area use only well-known technologies that are not subscriptionbased. For the second scenario (II in Figure 6(a)), two candidate networks are assumed to be unpopular to the shortrange neighbors. This corresponds to the setting discussed in Section 5.2 and is modeled by employing $v_{0}=0, v_{s}=0.1$, and $v_{h}=\left(1-2 v_{s}\right) /(N-2)$, where $v_{s}$ is the probability of unlikely networks and $v_{h}$ is the probability of networks that are likely to be preferred. In this case, it only makes sense to consider scenarios of at least $N=3$ networks (the 2 unpopular and at least one popular). Finally, in case III, which is similar to the one discussed in Section 5.3, we give a small probability to all the networks and let $v_{0}$ be close to 1 . This could be a scenario in which the MN has unusual wireless interfaces, for example, a WiMAX interface in an area where WiMAX is uncommon.

Energy results for the three scenarios and for the conventional VHO scheme are displayed in Figure 6(a) as a function of candidate networks $N$. As it can be observed, the average energy consumed in case I is up to $50 \%$ lower than that of the conventional scheme. An MN performing a handover in an environment with the characteristics of case I will most likely discover candidate networks through its shortrange neighbors, avoiding the costly scans of the conventional scheme. Moreover, we can see that as the number of candidate networks increases, the average energy consumption per handover gradually converges to a limiting value. This is because as the number of networks increases, the observed $\mathrm{MN}$ will probably manage to connect to one of the first few networks discovered using the short-range cooperation approach and thus no matter how many networks were actually discovered, no attempt of querying for resources or connecting will be made to most of them. This is in line with the discussion in Section 5.1 and the result in (16).

For case II, the short-range cooperation-assisted scheme performs marginally worse than in case I when the number of candidate networks is small but gets even as the number of networks increases. The energy consumption in this case is in accordance with the results of Section 5.2, which relate the consumption to the number of highly probable networks. Since we keep the number of networks that have low $v_{i}$ fixed, for a small total number of networks, the probability of discovering one of them through short-range cooperation will be low, forcing the MN most of the times to fall back to the conventional VHO scheme (and to higher energy consumption). However, as the number of networks increases, the highly probable networks become the majority, meaning that the likelihood of the MN discovering networks through short-range cooperation increases, reducing the probability that a costly scan will be required. Therefore, the existence of some unpopular networks becomes immaterial and the performance converges to that of scenario I.

Finally, for scenario III, the short-range cooperationassisted scheme exhibits lower performance, even worse than that of the conventional one. In the case where $v_{0}$ is close to 1 , (22) becomes almost equal to 1 and thus (12) yields $C_{\text {coop }} \approx$ $C_{\text {conv }}$. As a result, the cooperation-assisted scheme spends almost the same amount of energy as the conventional one for performing the actual handover procedure. However, the 


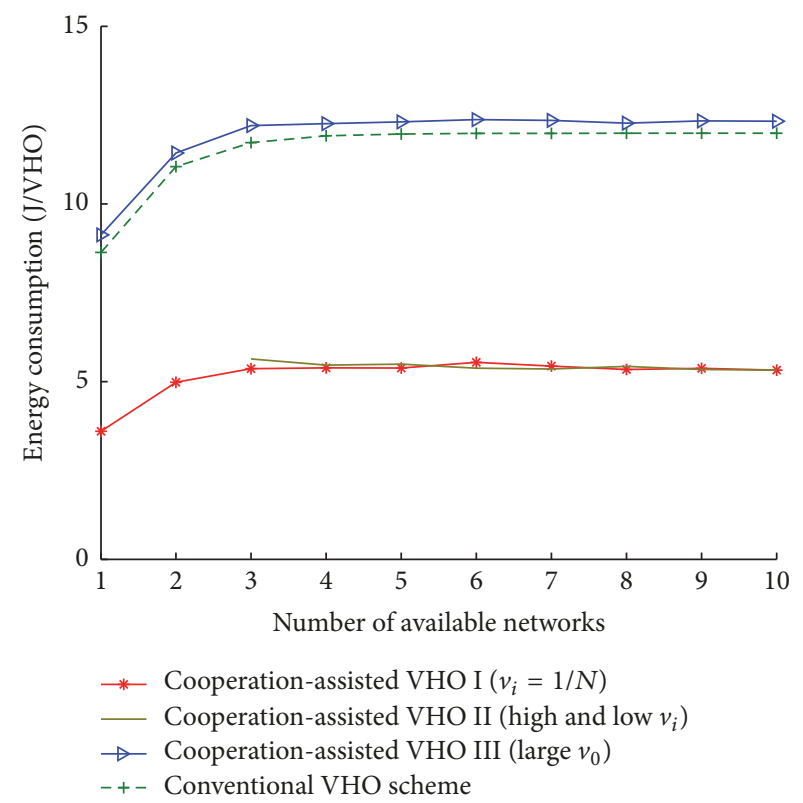

(a)

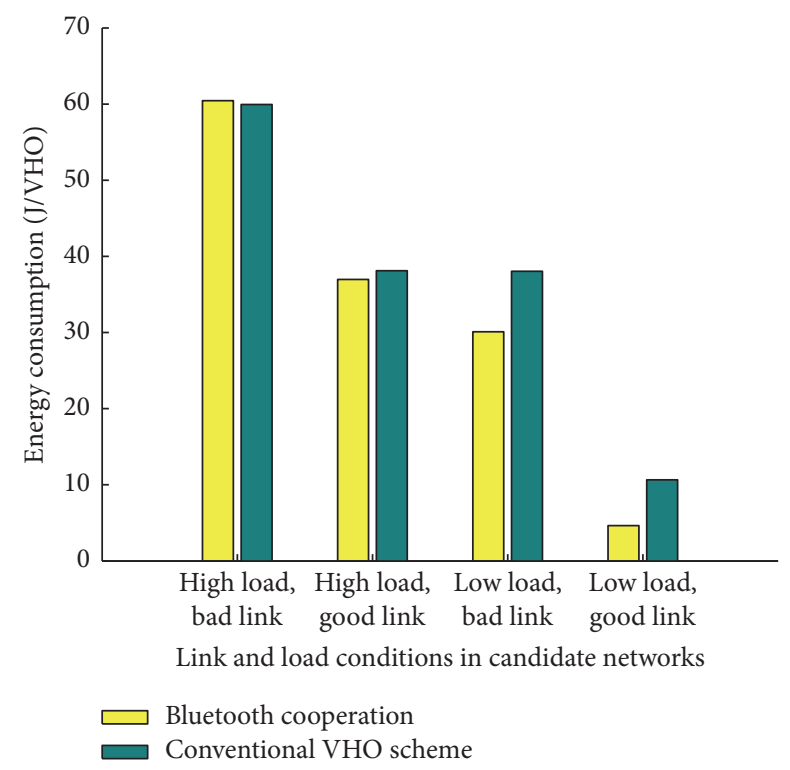

(b)

FIGURE 6: Average energy consumption of the cooperation-assisted VHO mechanism compared to that of the conventional VHO scheme in terms of the number of available networks and the network preference of the cooperating neighbors (a) and for various radio link and network load conditions (b).

cooperation scheme has the additional overhead of executing the short-range cooperation protocol and this leads to higher total energy consumption.

Turning to a different aspect, we now examine the performance of the $\mathrm{VHO}$ scheme as a function of the parameters related to the radio link conditions in the area (expressed through $p$ ) and the load of candidate networks (expressed through $q$ ) to the average energy consumption of both the conventional and the cooperation-assisted $\mathrm{VHO}$ schemes. We consider various combinations of the radio link conditions (good corresponds to 0.9 and bad to 0.2 ) and loading conditions at the candidate networks (high corresponds to 0.2 and low to 0.9 ). The results are shown in Figure 6(b).

We can observe that, in the first two cases where the load is high, the conventional scheme is almost on par with the cooperation-assisted one regardless of the link quality. On the other hand, once the network load becomes lower, the conventional cooperation-assisted scheme becomes more energy-efficient regardless of the radio link quality, indicating that the load parameter $q$ has greater impact on the cooperation-assisted scheme compared to the link quality. The reason for this is that while the network load conditions affect all aspects of the cooperation scheme, the effect of parameter $p$ is more limited, since no scanning is made before querying a candidate network for resources. Therefore $p$ has no impact in the association attempt to networks discovered through short-range cooperation. These results also align with the discussion made in Section 5.1, where it was seen that changes in the network load had a greater impact than changes in the link quality (quadratic effect of $q$ compared to linear effect of $p$ in the exponent of (17)).
To emphasize further this insight from Section 5, we turn to the final set of results in Figure 7 . In this, we measure the energy gain (as opposed to the absolute expenditure) per handover if we move from an environment with bad radio link conditions $(p=0.2)$ to an environment with good conditions $(p=0.9)$ for all possible network loads $(q \epsilon$ $(0,1])$. We compare this with the energy gain when moving from an environment with high network load $(q=0.2)$ to an environment with a low load $(q=0.9)$ for all possible radio link conditions $(p \in(0,1])$. As we can observe, when the network load drops, the gain is always higher than the gain we get when the radio link conditions improve.

\section{Implementation Aspects}

A basic requirement of the periodically-fully-awake-interval scheme employed in this work is the support for broadcasting messages. Even though the scheme, as explained in Section 3, is deliberately targeted to operate above the MAC layer, it is also important to see how the mechanism could be applied in various short-range radio access technologies. Therefore, in this section, we briefly discuss aspects related to the practical implementation of the short-range cooperation protocol, considering two wireless technologies that are currently available in virtually all commercial mobile devices: Bluetooth and Wi-Fi. Of course, a particular choice has implications (communication range and energy expenditure) that must be taken into account by appropriately tailored values of relevant parameters in the model presented in Section 4.

Bluetooth. While the conventional Bluetooth technology (usually referred to as Bluetooth 2.x) might be unsuitable for 


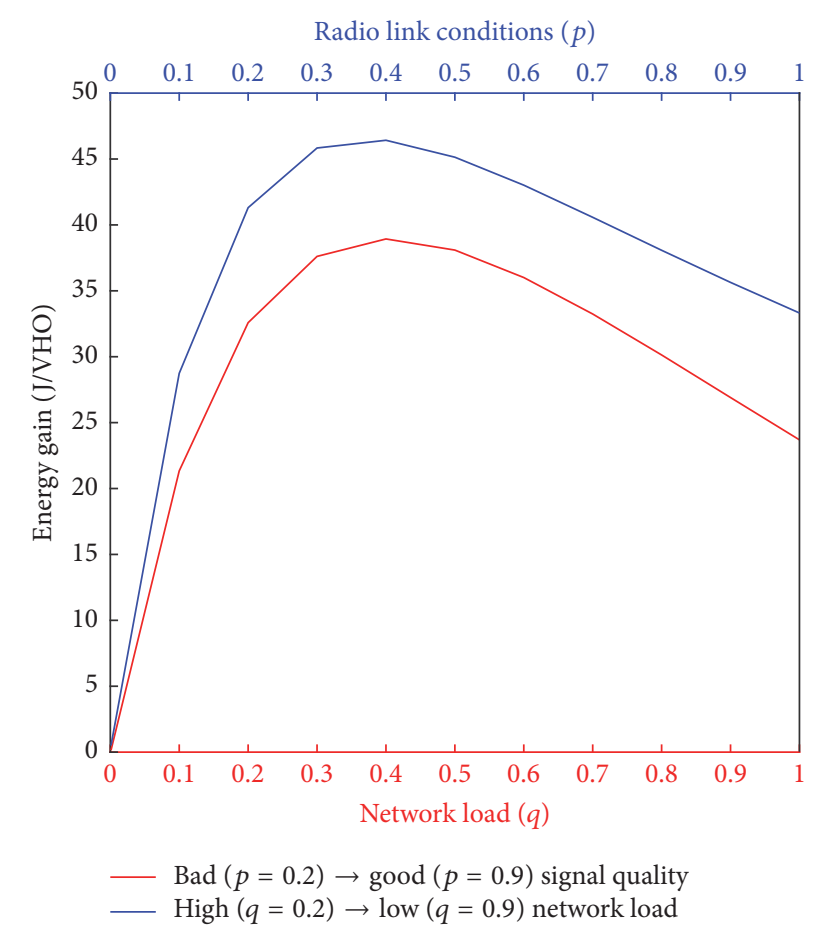

FIGURE 7: Effect of link and load conditions in the average energy consumption of the cooperation-assisted VHO mechanism.

use in the context of this work due to its inability to transmit data without pairing, newer Bluetooth versions inherently provide broadcasting support. More specifically, Bluetooth low energy (BLE) [22] provides a beaconing mechanism, which allows devices to broadcast short messages (31 bytes long). While the size of these beacons is not big enough to allow the transmission of all the network-related information required by an $\mathrm{MN}$, BLE provides a mechanism that allows a listener to request additional information from the broadcaster through follow-up messages. To further improve things, the more recent Bluetooth 5 [23] allows devices to broadcast larger messages (255 bytes), which means that all the relevant information about the available wireless network could be advertised in one go.

Wi-Fi. Broadcasting information related to the short-range protocol could be supported by $\mathrm{Wi}-\mathrm{Fi}$ in a number of ways. In the simplest case, an MN could broadcast networkrelated information during its AW period by turning into an AP and transmitting the information using appropriately formatted beacon frames. During the LW, it could turn back into a client and scan for available beacons. However, the problem with this approach is that (i) it can be very energy-consuming for the $\mathrm{MN}$ and (ii) makes the Wi-Fi interface unavailable for other uses. A more appropriate way of implementing the periodically-fully-awake-interval scheme would be by exploiting newer capabilities of Wi-Fi. For example, the network information could be broadcasted using Wi-Fi Direct [24] (e.g., Android's service discovery capabilities using its Wi-Fi P2P framework [25]) or using the Wi-Fi Aware standard [26], which allows mobile devices to discover, subscribe, and publish information to other neighboring devices.

\section{Concluding Remarks}

The paper introduced the concept of short-range cooperation for energy-efficient vertical handovers and developed a model for capturing the associated mean energy expenditure per handover by means of closed-form expressions. The model encapsulated various important cooperation and handover-related parameters and allowed the comparison of cooperation-assisted VHOs to the conventional handover scheme.

Simulation results validated the proposed analytical model and highlighted the energy efficiency benefits of the proposed scheme in various realistic scenarios. Additional aspects of the cooperation mechanism were evaluated through the analytical model and the effect of various relevant factors was studied. Results indicate network availability, radio link conditions in the local area, and network loading conditions in candidate networks to be the key influencing factors. The cost/benefit trade-offs of the short-range cooperation protocol were also studied, revealing that the cost can be nearly minimized through the proper parametrization of the protocol. Overall, the results show that the proposed cooperation scheme outperforms the conventional approach in terms of energy efficiency in several realistic usage scenarios.

The modeling methodology expresses per operation energy costs through appropriate coefficients. However, the proposed methodology could directly address mean handover latency instead of energy consumption, simply by using delay coefficients per operation, in place of the energy coefficients. For delay, the short-range coordination and status exchange cost is zero, this being a proactive background process without extra latency impact once the VHO is initiated.

As a final remark and given the character of shortrange communications, security-related issues, although out of this paper's scope, can be very significant for the practical applicability of the mechanism. They could be addressed by using a higher-layer trust-based mechanism to guard against malicious nodes. The model could compensate for this by adjusting the density of the spatial Poisson process to represent only the trustworthy nodes, for example, by a methodology similar to the one followed in [27].

As future work, we intend to make the cooperation mechanism adaptive to the surrounding conditions in order to further increase the energy efficiency of the proposed scheme. Depending on the density of neighboring nodes, an MN could adaptively choose to (de)activate the short-range cooperation protocol after considering the potential energy savings it could get from each configuration. For example, if an MN observes that it is located in a sparsely populated area (e.g., rural), where discovery of networks through the short-range cooperation mechanism is improbable, it should automatically deactivate the protocol and only check periodically to see whether the nodal density has changed making the reactivation of the protocol favorable. As another task for 
future work and given the observations of Sections 5 and 6 regarding the impact of the network load (expressed through parameter $q$ ) on the efficiency of the cooperation-assisted scheme, it would be interesting to investigate ways of capturing the variations in network load conditions at a finer scale.

\section{Appendix}

Here, we provide the proof of (1) for the conventional scheme's total mean energy cost per handover.

The information server is queried once, for the list of candidate networks, and this yields the first term in (1). Then, the $N$ candidate networks in the list are checked in turn. For each network, a scan is performed to determine the quality of the radio link from the MN to the network. If this quality is insufficient (with probability $1-p$ ), the next network in the list is processed. If the link quality is good (with probability $p$ ), the network will be queried for resources. If there are sufficient resources (with probability $q$ ), this network is selected as a handover target; otherwise the next network in the list is processed.

In view of the above, the total number of scans is equal to the total number of networks checked, which is itself equal to the number of independent Bernoulli trials with success probability $p q$, until the first success or until $N$ trials have been made. Therefore, the part of the cost related to scans is equal to

$$
\begin{gathered}
C_{\text {SCAN }}\left(\sum_{k=1}^{N} k p q(1-p q)^{k-1}+N(1-p q)^{N}\right) \\
=C_{\text {SCAN }} \frac{1-(1-p q)^{N}}{p q} .
\end{gathered}
$$

The second term inside the parentheses corresponds to the case of checking all $N$ candidate networks without success. The result above can be seen equal to the second term in (1).

Consider now the mean number of resource queries, conditional on the event that $k$ networks were checked in total, with the sequence ending with success. The last check was successful, so it necessarily included a resource query. Each of the preceding $k-1$ checks failed. This failure was due to a failed scan (with probability $(1-p) /(1-p q)$ ), taking into account the conditioning on the network check's overall failure), in which case no resource query was attempted, or due to a negative response to the resource query (this event occurring with the complementary probability $p(1-q) /(1-$ $p q)$ ). In view of these remarks, the conditional mean number of resource queries is equal to $(k-1) p(1-q) /(1-p q)+1$.

It is now straightforward to obtain the unconditional mean cost corresponding to resource queries as

$$
\begin{aligned}
C_{\mathrm{Q}}\left(\sum_{k=1}^{N}\left\{\left[(k-1) \frac{p(1-q)}{1-p q}+1\right] p q(1-p q)^{k-1}\right\}\right. \\
\left.+N \frac{p(1-q)}{1-p q}(1-p q)^{N}\right)=C_{\mathrm{Q}} p \\
+\frac{1-(1-p q)^{N}}{p q}
\end{aligned}
$$

and this is seen equal to the third term in (1). Note that the

\begin{tabular}{|c|c|}
\hline$p:$ & $\begin{array}{l}\text { Probability of sufficient radio link } \\
\text { quality }\end{array}$ \\
\hline$q:$ & $\begin{array}{l}\text { Probability of availability of network } \\
\text { resources }\end{array}$ \\
\hline$N:$ & Number of candidate networks \\
\hline$v_{i}, i=0 \cdots N:$ & $\begin{array}{l}\text { Network preferences of cooperating } \\
\text { peer }\end{array}$ \\
\hline$\sigma:$ & MNs per unit area \\
\hline$r:$ & $\begin{array}{l}\text { Communication range of short-range } \\
\text { technology }\end{array}$ \\
\hline$C_{\mathrm{IS}}, C_{\mathrm{SCAN}}, C_{\mathrm{Q}}:$ & $\begin{array}{l}\text { Cost for consulting the information } \\
\text { service, performing a scan, and } \\
\text { querying a network for resources }\end{array}$ \\
\hline$T:$ & $\begin{array}{l}\text { Average time between two consecutive } \\
\text { VHOs }\end{array}$ \\
\hline BI: & Beacon interval \\
\hline$j:$ & $\begin{array}{l}\text { Interval between two fully active } \\
\text { beacons }\end{array}$ \\
\hline$E_{\mathrm{AW}}, E_{\mathrm{LW}}, E_{\mathrm{IDLE}}:$ & $\begin{array}{l}\text { Total energy consumed during an AW, } \\
\text { LW, or idle period. }\end{array}$ \\
\hline
\end{tabular}
second term inside the outer parentheses in the left-hand side above corresponds to the event that all $N$ networks were checked unsuccessfully.

\section{Summary of Model Parameters}

\section{Conflicts of Interest}

The authors declare that there are no conflicts of interest regarding the publication of this paper.

\section{Acknowledgments}

This work is partly supported by the European FP7 project GREENET (FP7-PEOPLE-2010-ITN-264759).

\section{References}

[1] 3GPP, Architecture Enhancements for non-3GPP Accesses, vol. Rel-13 of 3GPP TS 23.402, 2015.

[2] IEEE 802.21 Working Group, IEEE Standard for Local and Metropolitan Area Networks - Part 21: Media Independent Handover, vol. 802, IEEE, 2009.

[3] N. Dimitriou, L. Sarakis, D. Loukatos, G. Kormentzas, and C. Skianis, "Vertical handover (VHO) framework for future collaborative wireless networks," International Journal of Network Management, vol. 21, no. 6, pp. 548-564, 2011.

[4] J. Soto, S. Queiroz, and M. Nogueira, "Managing sensing and cooperation to analyze PUE attacks in cognitive radio ad hoc networks," in Proceedings of the 2012 8th International Conference on Network and Service Management (CNSM '12), pp. 219-223, USA, October 2012.

[5] M. Papadopouli and H. Schulzrinne, "Effects of power conservation, wireless coverage and cooperation on data dissemination among mobile devices," in Proceedings of the 2001 ACM International Symposium on Mobile Ad Hoc Networking and Computing (MobiHoc '01), pp. 117-127, USA, October 2001. 
[6] A. Radwan and J. Rodriguez, "Energy saving in multi-standard mobile terminals through short-range cooperation," EURASIP Journal on Wireless Communications and Networking, vol. 2012, no. 1, 2012.

[7] H. Shan, W. Zhuang, and Z. Wang, "Distributed cooperative MAC for multihop wireless networks," IEEE Communications Magazine, vol. 47, no. 2, pp. 126-133, 2009.

[8] J. Alonso-Zárate, E. Kartsakli, C. Verikoukis, and L. Alonso, "Persistent RCSMA: A MAC protocol for a distributed cooperative ARQ scheme in wireless networks," EURASIP Journal on Advances in Signal Processing, vol. 2008, no. 1, Article ID 817401, 2008.

[9] J. Alonso-Zárate, L. Alonso, and C. Verikoukis, "Performance analysis of a persistent relay carrier sensing multiple access protocol," IEEE Transactions on Wireless Communications, vol. 8, no. 12, pp. 5827-5831, 2009.

[10] J. M. Marquez-Barja, H. Ahmadi, S. M. Tornell et al., "Breaking the vehicular wireless communications barriers: Vertical handover techniques for heterogeneous networks," IEEE Transactions on Vehicular Technology, vol. 64, no. 12, pp. 5878-5890, 2015.

[11] D. A. Wassie, D. Loukatos, L. Sarakis, K. Kontovasilis, and C. Skianis, "On the energy requirements of vertical handover operations: Measurement-based results for the IEEE 802.21 framework," in Proceedings of the 2012 IEEE 17th International Workshop on Computer Aided Modeling and Design of Communication Links and Networks (CAMAD '12), pp. 145-149, Spain, September 2012.

[12] X. Foukas, K. Kontovasilis, and M. K. Marina, "Exploiting short-range cooperation for energy efficient vertical handover operations," in Proceedings of the 11th International Conference on Network and Service Management (CNSM '15), pp. 292-300, IEEE, Barcelona, Spain, November 2015.

[13] S. Park and S.-M. Yoo, "An efficient reliable one-hop broadcast in mobile ad hoc networks," Ad Hoc Networks, vol. 11, no. 1, pp. 19-28, 2013.

[14] Y. Agarwal, C. Schurgers, and R. Gupta, "Dynamic power management using on demand paging for networked embedded systems," in Proceedings of the 2005 Asia and South Pacific Design Automation Conference (ASP-DAC '05), pp. 755-759, IEEE, Shanghai, China, January 2005.

[15] J. Wu and F. Dai, "Efficient broadcasting with guaranteed coverage in mobile ad hoc networks," IEEE Transactions on Mobile Computing, vol. 4, no. 3, pp. 259-270, 2005.

[16] R. Mahjourian, M. Thai, F. Chen, H. Zhai, R. Tiwari, and Y. Fang, "An approximation algorithm for conflict-aware broadcast scheduling in wireless ad hoc networks," in Proceedings of the 9th ACM International Symposium on Mobile Ad Hoc Networking and Computing (MobiHoc '08), pp. 331-340, May 2008.

[17] Y.-C. Tseng, C.-S. Hsu, and T.-Y. Hsieh, "Power-saving protocols for IEEE 802.11-based multi-hop ad hoc networks," Computer Networks, vol. 43, no. 3, pp. 317-337, 2003.

[18] J. S. Lee, Y. W. Su, and C. C. Shen, "A comparative study of wireless protocols: bluetooth, UWB, ZigBee, and Wi-Fi," in Proceedings of the 33rd Annual Conference of the IEEE Industrial Electronics Society (IECON '07), pp. 46-51, Taipei, Taiwan, November 2007.

[19] "NS-3 Log distance propagation model," https://www.nsnam .org/doxygen/classns3_1_1_log_distance_propagation_loss _model.htmlNS-3Logdistancepropagationmodel.
[20] “NS-3 802.11 PHY layer model," https://www.nsnam .org/doxygen/classns3_1_1_yans_wifi_phy.htmlNS-3802. 11PHYlayermodel.

[21] G. Auer, V. Giannini, I. Gódor et al., "Cellular energy efficiency evaluation framework," in Proceedings of the 2011 IEEE 73rd Vehicular Technology Conference (VTC '11-Spring), pp. 1-6, May 2011.

[22] Bluetooth SIG, "Bluetooth core specification version 4.2," Specification of the Bluetooth System, 2014.

[23] Bluetooth SIG, "Bluetooth core specification version 5.0," Specification of the Bluetooth System, 2016.

[24] D. Camps-Mur, A. Garcia-Saavedra, and P. Serrano, "Deviceto-device communications with WiFi direct: overview and experimentation," IEEE Wireless Communications Magazine, vol. 20, no. 3, pp. 96-104, 2013.

[25] Google, "Wi-Fi Peer-to-Peer," 2017-08-28, https://developer .android.com/guide/topics/connectivity/wifip2p.html.

[26] Wi-Fi Alliance, "Wi-Fi Aware," 2017-08-28, https://www.wi-fi .org/discover-wi-fi/wi-fi-aware.

[27] I. Manolopoulos, K. Kontovasilis, I. Stavrakakis, and S. C. A. Thomopoulos, "Exploiting topology and behavioral attributes for effective routing in mobile networks," in Proceedings of the 2013 10th Annual Conference on Wireless On-Demand Network Systems and Services (WONS '13), pp. 101-103, Canada, March 2013. 


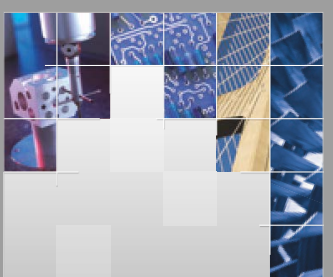

\section{Enfincering}
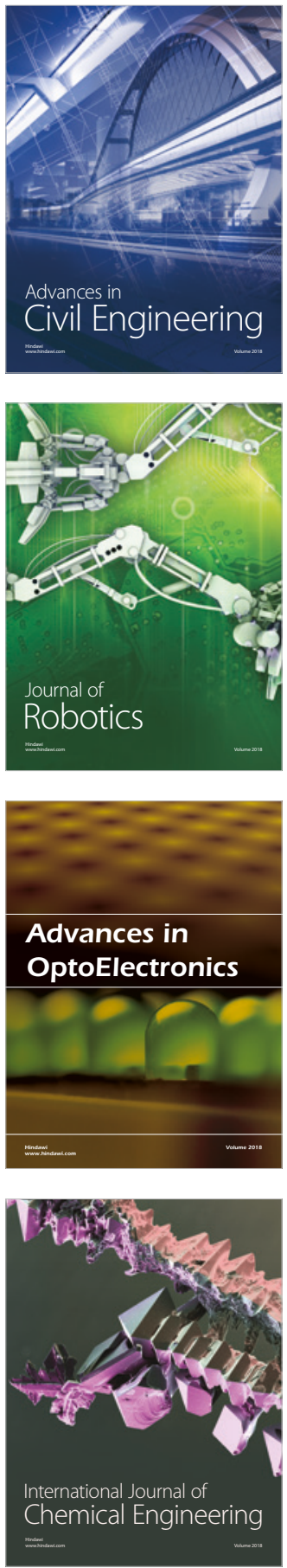

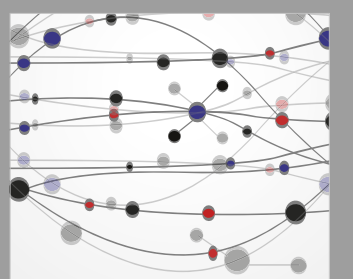

\section{Rotating \\ Machinery}

The Scientific World Journal

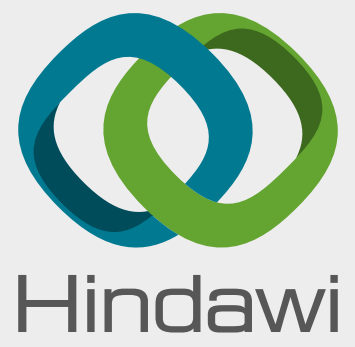

Submit your manuscripts at

www.hindawi.com
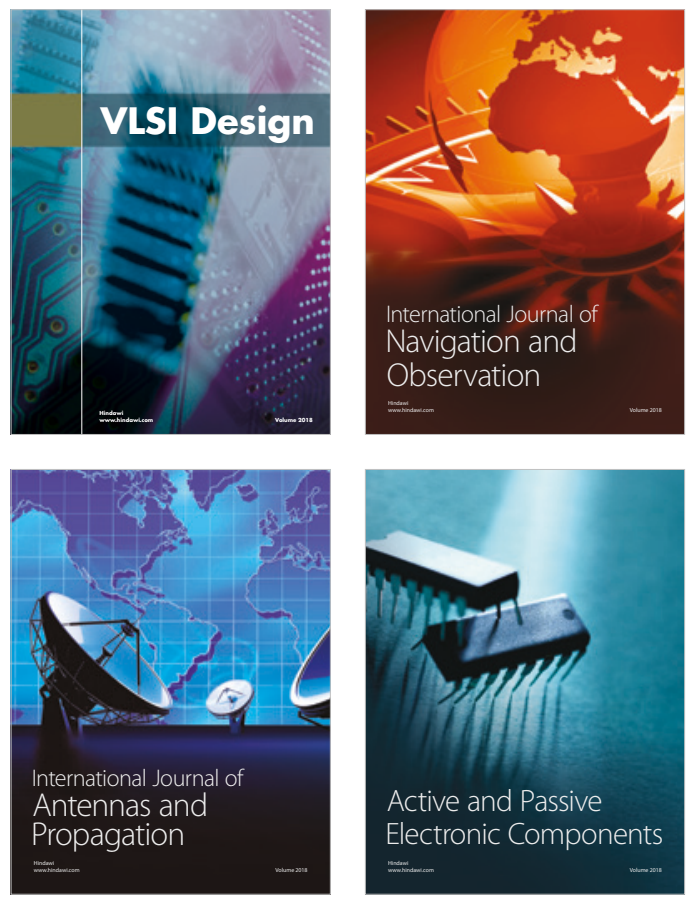
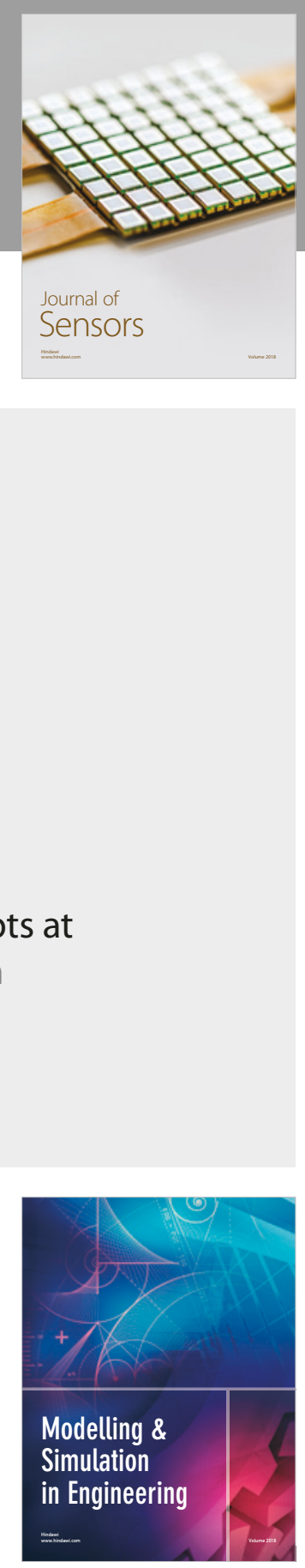

\section{Advances \\ Multimedia}
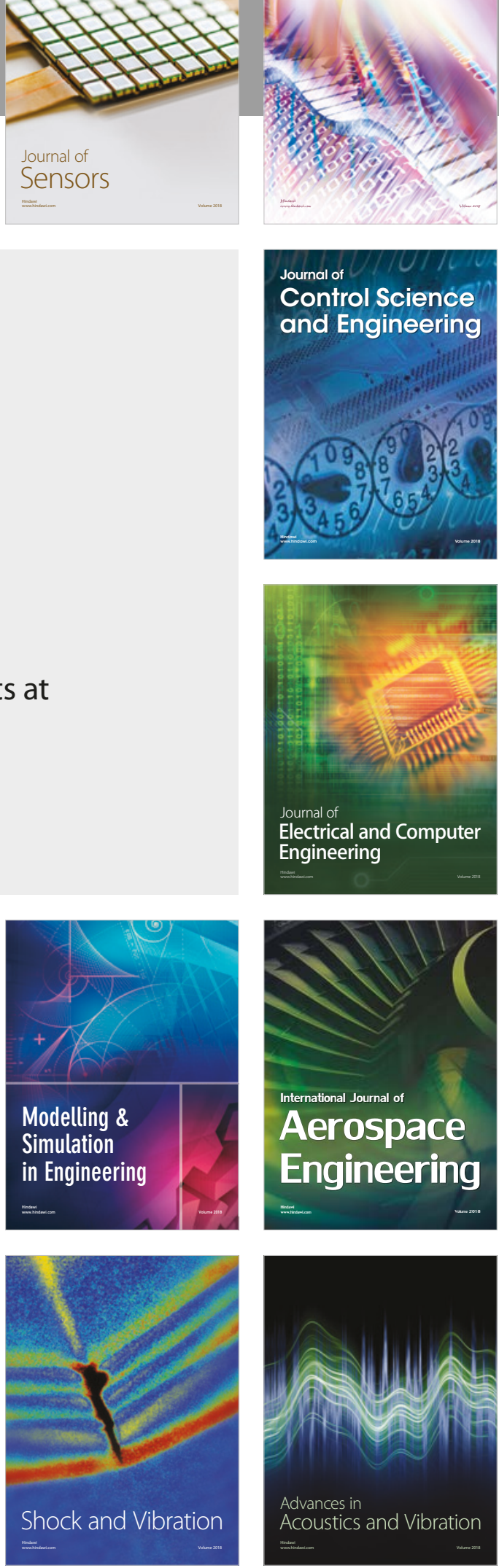